\title{
Can we understand an auxetic pion-photon transition form factor within QCD? BaBar faces Belle*
}

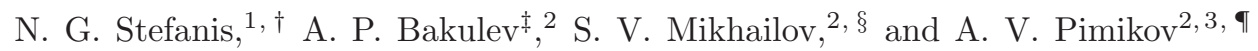 \\ ${ }^{1}$ Institut für Theoretische Physik II, Ruhr-Universität Bochum, D-44780 Bochum, Germany \\ ${ }^{2}$ Bogoliubov Laboratory of Theoretical Physics, JINR, 141980 Dubna, Russia \\ ${ }^{3}$ Departamento de Física Teórica-IFIC, Universidad de Valencia-CSIC, E-46100 Burjassot (Valencia), Spain
}

(Dated: November 9, 2018)

\begin{abstract}
A state-of-the-art analysis of the pion-photon transition form factor is presented based on an improved theoretical calculation that includes the effect of a finite virtuality of the quasi-real photon in the method of light-cone sum rules. We carry out a detailed statistical analysis of the existing experimental data using this method and by employing pion distribution amplitudes with up to three Gegenbauer coefficients $a_{2}, a_{4}, a_{6}$. Allowing for an error range in the coefficient $a_{6} \approx 0$, the theoretical predictions for $\gamma^{*} \gamma \rightarrow \pi^{0}$ obtained with nonlocal QCD sum rules are found to be in good agreement with all data that support a scaling behavior of the transition form factor at higher $Q^{2}$, like those of the Belle Collaboration. The data on $\gamma^{*} \gamma \rightarrow \eta / \eta^{\prime}$ from CLEO and BABAR are also reproduced, while there is a strong conflict with the auxetic trend of the BABAR data above $10 \mathrm{GeV}^{2}$. The broader implications of these findings are discussed.
\end{abstract}

PACS numbers: 12.38.Lg, 12.38.Bx, 13.40.Gp, 11.10.Hi

\section{INTRODUCTION}

The data of the BABAR Collaboration [1] of the $\gamma^{*}\left(q_{1}^{2}\right) \gamma\left(q_{2}^{2}\right) \rightarrow \pi^{0}$ (with $q_{1}^{2}=-Q^{2}$ for the far off the mass shell photon and $q_{2}^{2}=-q^{2} \approx 0$ for the near on mass shell photon) transition form factor (TFF) in the wide momentum-transfer range from 4 to $40 \mathrm{GeV}^{2}$ have not yet found a satisfactory explanation within the (collinear) factorization approach of QCD. As first pointed out in [2], the rise of the scaled form factor $Q^{2} F^{\gamma^{*} \gamma \pi^{0}}\left(Q^{2}\right) \equiv$ $\mathcal{F}^{\gamma \pi}\left(Q^{2}\right)$ observed by $B A B A R$ above $10 \mathrm{GeV}^{2}$ up to the highest momentum probed (with the exception of two data points at about 14 and $27 \mathrm{GeV}^{2}$ that are below and close to the asymptotic limit $\mathcal{F}_{Q^{2} \rightarrow \infty}^{\gamma \pi}\left(Q^{2}\right)=\sqrt{2} f_{\pi} \mathrm{GeV}$ with $f_{\pi} \approx 0.131 \mathrm{GeV}$ ) does not conform with the standard QCD approach based on collinear factorization - see [3] for a review.

Following the publication of the BABAR data on $\mathcal{F}^{\gamma \pi}\left(Q^{2}\right)$ in 2009 there was a spurt of worldwide theoretical activity, using different approaches and drawing strongly diverging conclusions (see [4] for the original analysis and [5] for a brief benchmark comparison with other approaches). For instance, Agaev et al. [6] claimed that the BABAR data for the pion-photon transition form factor (TFF) can be accommodated within QCD using light-cone sum rules (LCSR)s in conjunction with pion distribution amplitudes (DA)s characterized by an inverse hierarchy of Gegenbauer coefficients $a_{4}>a_{2}$ (and

\footnotetext{
* This work is dedicated to the memory of Klaus Goeke- friend and colleague.

$\ddagger$ deceased

${ }^{\dagger}$ Electronic address: stefanis@tp2.ruhr-uni-bochum.de

$\S$ Electronic address: mikhs@theor.jinr.ru

๑Electronic address: alexandr.pimikov@uv.es
}

eventually including still higher terms $\left.a_{6}, a_{8}, a_{10}, \ldots\right)$. In contrast, our recent analysis in [4] - which utilizes basically the same method but in connection with endpointsuppressed pion DAs [7] based solely on $a_{2}, a_{4}$ — comes to the opposite conclusion.

In 2012, the Belle Collaboration [8] reported upon a new measurement of the process $\gamma^{*} \gamma \rightarrow \pi^{0}$ at the KEKB collider for the kinematical region $4 \mathrm{GeV}^{2} \lesssim Q^{2} \lesssim$ $40 \mathrm{GeV}^{2}$. The main message from this new experiment is that the measured values of $\mathcal{F}^{\gamma \pi}\left(Q^{2}\right)$ agree with the previous measurements of CELLO [9] and CLEO [10] and also with the data of $B A B A R$ in the momentum range $Q^{2} \lesssim 9 \mathrm{GeV}^{2}$, while at still higher momenta they do not show a growth with $Q^{2}$ but are more or less close to the asymptotic limit of QCD with the exception of a single point that gives a larger value of $\mathcal{F}^{\gamma \pi}\left(Q^{2}\right)$. Surprisingly, this outlier at $27 \mathrm{GeV}^{2}$ shows exactly the opposite behavior relative to the BABAR measurement at the same momentum value that coincides with the asymptotic limit.

Such an incongruent behavior of the data does not allow a unique theoretical description, because there is no characteristic mathematical signature which emerges from the statistics of these measurements that would allow to draw reliable conclusions about the size of the scaled TFF at large $Q^{2}$. This issue was pondered in our recent paper in [11], in which we proposed a basic classification scheme of the available data juxtaposed with the cutting-edge theoretical predictions from various approaches. Referring to the scaled TFF vs. $Q^{2}$ (see Fig. 2 in [1]), this scheme consists of two distinct bands - one exhibiting scaling at high $Q^{2}$ (Belle data), the other sloping upward (BABAR data), while there is a third band in between collecting some theoretical results that are indifferent. Pooling all data in a single database (see Fig. 3 in [11]), one faces the problem that the underlying theoretical approaches are hardly compatible to each other because they correspond to very different underly- 
ing mechanisms.

These issues were further investigated in [12, 13], where we performed a two- and a three-parameter fit to all data utilizing, correspondingly, the Gegenbauer coefficients $a_{2}, a_{4}$ and $a_{2}, a_{4}, a_{6}$ within LCSRs. The main conclusion from these studies is that both fits to the combined sets of the data from CELLO, CLEO, and Belle (termed $\mathrm{CCBe}$ ), on one hand, and the set consisting of the CELLO, CLEO, and BABAR data (termed CCBB), on the other, have no overlap. The CCBe band indicates scaling, as predicted by QCD, while the CCBB band exhibits an auxetic 68] behavior that cannot be accommodated within the standard QCD scheme of collinear factorization. This finding reinforces our previous results in 11], favoring the classification pattern of two distinct bands rather than a single one that encompasses all data. Moreover 12, 13], the CCBe data set supports the theoretical predictions derived with the help of LCSRs in [14, 15] using a pion DA (termed BMS) extracted before from QCD sum rules with nonlocal condensates in [7]-see also [16, 17]. Indeed, the BMS $\pi^{0} \mathrm{DA}$ fits the CCBe data in terms of $a_{2}$ and $a_{4}$ with an accuracy of $\chi_{\text {ndf }}^{2}=\chi^{2} /$ ndf $=22.1 / 33$, where $\mathrm{ndf}=$ number of degrees of freedom.

Moreover, the same calculation [4] agrees with the BABAR data [18] for the processes $\gamma^{*} \gamma \rightarrow \eta, \gamma^{*} \gamma \rightarrow \eta^{\prime}$ using the description of the $\eta-\eta^{\prime}$ mixing in the quark flavor basis [19] to relate the form factor of the $|n\rangle=$ $(1 / \sqrt{2})(|u \bar{u}\rangle+|d \bar{d}\rangle)$ state to that of the pion. An immediate implication of this agreement is that the DA of the nonstrange component $|n\rangle$ of the $\eta, \eta^{\prime}$ mesons should be similar in shape to that of the $\pi^{0}$. This would mean in turn that there should be no strong flavor symmetry breaking in the pseudoscalar meson sector of QCD. On the other hand, the CCBB data set does not support the BMS-type of DAs, that have their endpoints suppressed, and demands the inclusion of at least the next coefficient $a_{6}$, or even higher ones, as proposed in [6]. Best agreement with the CCBB set is, however, provided with flat-type DAs 20, 21] which, at the same time, fail to reproduce the $\mathrm{CCBe}$ data.

In this work, we extend and refine our data analysis for $\mathcal{F}^{\gamma \pi}\left(Q^{2}\right)[2,4,11,14,15,22$, 23] (consult also [6, 24]) in the following points: (i) We estimate quantitatively the theoretical uncertainty owing to the small virtuality of the quasi-real photon in an attempt to take into account the unknown dependence on the momentum transfer to the untagged electron. 69] To this end, we define a new quantity $\Delta\left(Q^{2}\right)$ that "measures" the susceptibility of the TFF to the variations of $q^{2}$. (ii) We extend the original BMS DA "bunch" by considering the correlated "noise" related to the coefficient $a_{6}$ [7] and allowing it to vary with an appropriate error rate. (iii) We consider in detail the statistical properties of the BABAR and the Belle data and discuss the features of the statistical fluctuations and their influence on the $Q^{2}$-behavior of the pionphoton TFF in terms of two different fit models used in the literature. (iv) We give a qualitative discussion of the spacelike TFFs $Q^{2} F^{\gamma^{*} \gamma \eta\left(\eta^{\prime}\right)}\left(Q^{2}\right) \equiv \mathcal{F} \gamma \eta\left(\eta^{\prime}\right)\left(Q^{2}\right)$ in comparison with the recent data of BABAR [18] and the older ones of CLEO [10].

The paper is organized as follows: In Sec. II we sketch the theoretical scheme used in this work. In Sec. III we present a theoretical tool to probe the sensitivity of the TFF to the small photon virtuality. The inclusion of the correlated noise owing to the coefficient $a_{6}$ is discussed in the same section. Section IV is devoted to the statistical analysis of the various data sets relative to each other and against theoretical predictions. Section $\mathrm{V}$ contains an in-depth discussion of our results, while our conclusions are drawn in Sec. VI Important technical details are collected in three appendices.

\section{THEORETICAL BASIS}

The pion-photon TFF $F^{\gamma^{*} \gamma^{*} \pi^{0}}$ is described by the following matrix element

$$
\begin{gathered}
\int d^{4} z e^{-i q_{1} \cdot z}\left\langle\pi^{0}(P)\left|T\left\{j_{\mu}(z) j_{\nu}(0)\right\}\right| 0\right\rangle=i \epsilon_{\mu \nu \alpha \beta} q_{1}^{\alpha} q_{2}^{\beta} \\
\times F^{\gamma^{*} \gamma^{*} \pi^{0}}\left(Q^{2}, q^{2}\right)
\end{gathered}
$$

where $j_{\mu}$ is the quark electromagnetic current. For a faroff-shell photon with the momentum $Q^{2}$ and an almost real photon with the small virtuality $q^{2}$ the TFF within the method of LCSRs is given by the expression

$$
Q^{2} F^{\gamma^{*} \gamma^{*} \pi}\left(Q^{2}, q^{2}\right)=\frac{\sqrt{2}}{3} f_{\pi}\left[\frac{Q^{2}}{m_{\rho}^{2}+q^{2}} \int_{x_{0}}^{1} \exp \left(\frac{m_{\rho}^{2}-Q^{2} \bar{x} / x}{M^{2}}\right) \bar{\rho}\left(Q^{2}, x\right) \frac{d x}{x}+\int_{0}^{x_{0}} \bar{\rho}\left(Q^{2}, x\right) \frac{Q^{2} d x}{\bar{x} Q^{2}+x q^{2}}\right]
$$

Here $x$ denotes the longitudinal momentum fraction carried by the struck parton, $\bar{x}=1-x$ is the momentum of the spectator, $x_{0}=\left(Q^{2}\right) /\left(Q^{2}+s_{0}\right), s=\bar{x} Q^{2} / x, M^{2}$ is the Borel parameter, and the spectral density has the

$$
\begin{aligned}
& \text { form } \bar{\rho}\left(Q^{2}, x\right)=\left(Q^{2}+s\right) \rho^{\text {pert }}\left(Q^{2}, s\right), \text { where } \\
& \rho^{\text {pert }}\left(Q^{2}, s\right)=\frac{1}{\pi} \operatorname{Im} F^{\gamma^{*} \gamma^{*} \pi^{0}}\left(Q^{2},-s-i \varepsilon\right) \\
& =\rho_{\mathrm{tw}-2}+\rho_{\mathrm{tw}-4}+\rho_{\mathrm{tw}-6}+\ldots .
\end{aligned}
$$



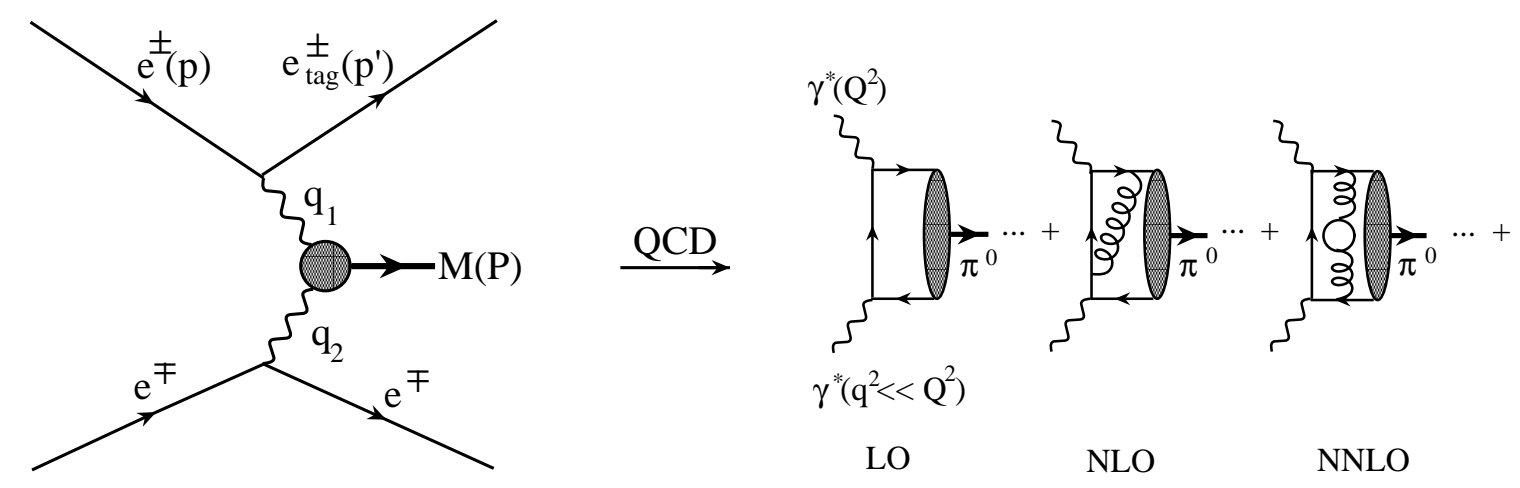

Figure 1: Left. Generic experimental setup for the process $e^{+} e^{-} \rightarrow e^{+} e^{-} M$, where $M$ is a pseudoscalar meson $\pi^{0}, \eta$ or $\eta^{\prime}$. The tagged electron (or positron) is labeled and the corresponding momenta of all particles are denoted. Right. Basic diagrams describing the process within QCD on the basis of collinear factorization order by order of perturbation theory: leading order (LO), next-to-leading order (NLO), and next-to-next-to-leading order (NNLO).

Each term of definite twist (abbreviated by tw) is calculable with the help of the analogous hard part convoluted with the pion distribution amplitude of the same twist
[25].

The Taylor expansion of the TFF with respect to $q^{2}$ to order $\delta q^{2}$ reads

$$
\left.Q^{2} F^{\gamma^{*} \gamma \pi}\left(Q^{2}, q^{2}\right)\right|_{q^{2}=0}+\left.Q^{2} \frac{d}{d q^{2}} F^{\gamma^{*} \gamma \pi^{0}}\left(Q^{2}, q^{2}\right)\right|_{q^{2}=0} \delta q^{2} \equiv \mathcal{F}^{\gamma \pi}\left(Q^{2}\right)+\left(\mathcal{F}^{\gamma \pi}\right)_{q^{2}=0}^{\prime}\left(Q^{2}\right) \delta q^{2}
$$

The first term in (4) is the usual expression obtained with a strictly real photon, whereas the second one gives the first non-vanishing contribution of a finite virtuality. The sum of the twist-two and the twist-four spectral densities, $\bar{\rho}\left(Q^{2}, x\right)$, as well as the explicit form of the terms on the r.h.s. of Eq. (4) are provided in Appendices $\mathrm{A}$ and $\mathrm{B}$, respectively. In this work, the twistfour term is taken into account in the effective form 25] $\varphi_{\pi}^{(4)}\left(x, \mu^{2}\right) \sim \delta_{\mathrm{tw}-4}^{2}\left(\mu^{2}\right) x^{2}(1-x)^{2}$. As usual in LCSR calculations, $s_{0} \simeq 1.5 \mathrm{GeV}^{2}$ is the effective threshold in the vector channel.

At the level of twist-two, the TFF has the following expansion

$$
\begin{aligned}
F_{\gamma^{*} \gamma^{*} \pi^{0}}^{\mathrm{tw}-2} \sim & {\left[T_{\mathrm{LO}}+a_{s}\left(\mu^{2}\right) T_{\mathrm{NLO}}+a_{s}^{2}\left(\mu^{2}\right) T_{\mathrm{NNLO}_{\beta_{0}}}+\ldots\right] } \\
& \otimes \varphi_{\pi}^{(2)}\left(x, \mu^{2}\right)
\end{aligned}
$$

in which the leading- (LO), next-to-leading (NLO), and next-to-next-to-leading (NNLO) terms are displayed. The corresponding Feynman graphs are depicted in Fig. 1. Note that our calculations here are incorporating the NLO spectral density in the corrected form pointed out in [6]. The nonperturbative content of the TFF is encoded in the pion DA. In our previous analysis in [4, 11], we have considered several proposed models for $\varphi_{\pi}^{(2)}$ and have compared the predictions extracted from them with all existing experimental data. For the scope of the present analysis, it is sufficient to employ only the "bunch" of twist-two pion DAs determined in 7] within the framework of QCD sum rules (SR) with nonlocal condensates (NLC). This DA "bunch" can be effectively parameterized in terms of the first two Gegenbauer coefficients $a_{2}$ and $a_{4}$, so that one has $(\xi \equiv x-\bar{x})$

$$
\varphi_{\pi}^{\mathrm{BMS}}(x)=6 x \bar{x}\left[1+a_{2} C_{2}^{3 / 2}(\xi)+a_{4} C_{4}^{3 / 2}(\xi)\right],
$$

whereas all higher coefficients have also been determined but found to be negligible, albeit with relatively large uncertainties, so that they were ignored. The DA with the coefficients $a_{2}\left(\mu^{2}\right)=0.20$ and $a_{4}\left(\mu^{2}\right)=-0.14$ (at $\mu^{2}=1 \mathrm{GeV}^{2}$ ) is termed the BMS model (from Bakulev, Mikhailov, Stefanis) and is marked out by the fact that its first ten moments $\left\langle\xi^{N}\right\rangle_{\pi} \equiv \int_{0}^{1} d x(2 x-1)^{N} \varphi_{\pi}^{(2)}\left(x, \mu^{2}\right)$ with the normalization condition $\int_{0}^{1} d x \varphi_{\pi}^{(2)}\left(x, \mu^{2}\right)=1$ lie within a particular range of values computed in [7]. The connection between the moments $\left\langle\xi^{N}\right\rangle_{\pi}$ and the coefficients $a_{2 n}$ is outlined in Appendix C Covering the whole admissible set of $\left\{a_{2}, a_{4}\right\}$ values gives rise to the "BMS bunch" of pion DAs. The extension of this "bunch" to a $3 \mathrm{D}$ set of coefficients $\left\{a_{2}, a_{4}, a_{6}\right\}$ will be considered further below.

The key characteristic of the BMS $\pi^{0}$ DAs is that their kinematic endpoints $x=0,1$ are strongly suppressed. This suppression is related to the assumption that the vacuum quarks have a non-zero virtuality $\lambda_{q}^{2}=$ $\left\langle\bar{q}\left(i g G_{\mu \nu} \sigma_{\mu \nu}\right) q\right\rangle / 2\langle\bar{q} q\rangle \simeq(0.35-0.55) \mathrm{GeV}^{2}$, pertaining 
to the use of QCD SRs with NLCs [7, 16, 17]. This is an approach rooted in the hypothesis that in the coordinate representation the NLCs are not constant but depend on the Euclidean separation of the quark fields and decay with a correlation length $\Lambda_{\text {corr }} \sim 1 / \lambda_{q}$-see [26, 27] for more details and [28] for related explanations. Then, the endpoint contributions in the scalar condensate that dominates the pion sum rule are strongly suppressed -in contrast to the standard approach of Chernyak and Zhitnitsky (CZ) 29], in which just these regions dominate the $\pi^{0} \mathrm{DA}$.

It turns out [4, 11] that the majority of the existing experimental data at intermediate $Q^{2} \leq 9 \mathrm{GeV}^{2}$ are best described by such endpoint-suppressed pion DAs [7]. This becomes obvious from Fig. 2 (upper green band), where we compare our predictions with various experimental data using for the horizontal axis a logarithmic scale in order to "stretch out" the smaller values of $Q^{2}$. In particular the Belle data are in good agreement with our predictions up to $40 \mathrm{GeV}^{2}$, whereas the high- $Q^{2}$ tail of the $\pi^{0} B A B A R$ data is in conflict with these predictions.

\section{METHODS OF DATA ANALYSIS}

\section{A. Small virtuality of quasi-real photon}

In the first part of this section we address the treatment of a small but finite virtuality of the quasi-real photon within the method of LCSRs.

As we announced in the Introduction and expounded in Sec. [II] we include into our calculation of the $\pi$ TFF the small virtuality of the quasi-real photon in an attempt to mimic the real situation of a single-tag experiment, like that of $B A B A R$ and Belle. Such experiments bear an uncertainty owing to the unknown dependence on the momentum transfer to the untagged electron. This means that the facility can register only events with a momentum of the quasi-real photon $\left|q^{2}\right|$ up to some limiting value. To get an estimate of this effect on the calculation, we use for our numerical evaluation the area of photon momenta probed in the Belle experiment [8] and allow a variation of $q^{2}$ in the range $q^{2} \lesssim 0.04 \mathrm{GeV}^{2}$ down to $0.01 \mathrm{GeV}^{2}[70]$. This area is compatible with the cuts imposed by CLEO which accept two charged tracks, each of transverse momentum of the order of $\delta q^{2} \simeq 0.01 \mathrm{GeV}^{2}$, while $B A B A R$ [1] imposed an upper limit of $\left|q_{2}^{2}\right|<0.18 \mathrm{GeV}^{2}$. Employing Eqs. (2) and (A2), we compute the TFF and present the result graphically in Fig. 2 in terms of the lower narrow (red) strip that enlarges the original BMS green band downwards.

The other key ingredients of our LCSR calculation in this work are the following: QCD radiative corrections with NLO accuracy and the twist-four contribution are explicitly included. The main NNLO term, proportional to $\beta_{0}$ [30], is taken into account implicitly by means of uncertainties together with the twist-six term calculated in [6]. Note that the $\mathrm{NLO}, \mathrm{NNLO}_{\beta}$, and twist-

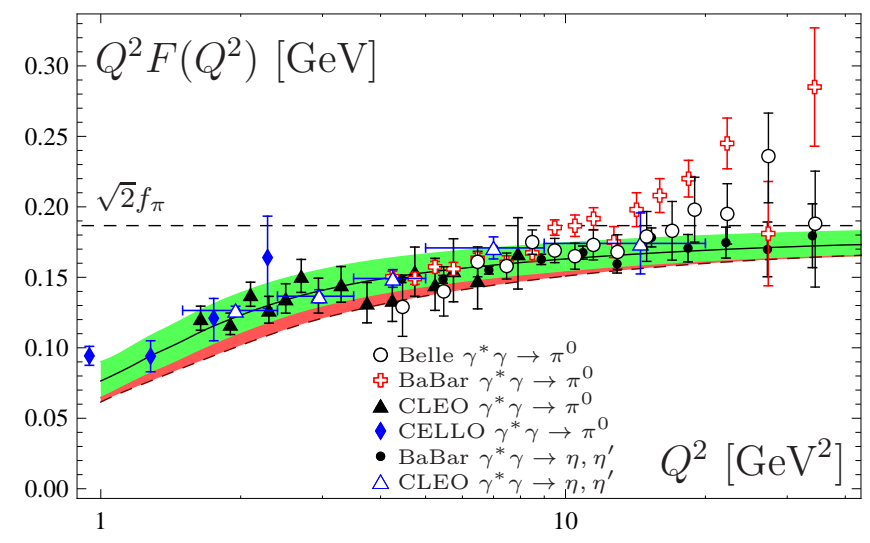

Figure 2: (color online). Logarithmic plot of the theoretical predictions for the scaled $\gamma^{*} \gamma \pi^{0}$ transition form factor in comparison with data taken from various experiments, as indicated. The upper (green) band shows the results obtained within our approach [4, 11] assuming that the quasi-real photon has vanishing virtuality. The lower (red) strip represents the influence on the TFF of the small virtuality of the quasireal photon induced by the untagged electron in the Belle experiment $\left(q^{2} \approx 0.04 \mathrm{GeV}^{2}\right)$.

four contribution are all negative, supplying suppression, whereas the twist-six term has a positive sign and is either very small if a Borel parameter of $M^{2}=1.5 \mathrm{Gev}^{2}$ is used-as in [6] - or it has for $M^{2}$ varying in the interval $M^{2} \in[0.7 ; 0.9] \mathrm{GeV}^{2}$ —as in [4, 11] - approximately the same size as the $\mathrm{NNLO}_{\beta}$ radiative correction and almost cancels against it. It is worth mentioning that the Borel parameter in our approach is not fixed to a particular value, but is allowed to vary with $Q^{2}$ according to the relation $M^{2}=M_{2 \text {-pt }}^{2} /\langle x\rangle\left(Q^{2}\right)$, i.e., becoming smaller with increasing $Q^{2}$. Here $M_{2-\mathrm{pt}}^{2}$ is the two-point Borel parameter that is specified in the two-point QCD SR for the $\rho$-meson at the mean value $M_{2 \text {-pt }}^{2}=0.7 \mathrm{GeV}^{2}$, while $\langle x\rangle\left(Q^{2}\right)$ is some average value of $x$ at fixed value of $Q^{2}$. We emphasize that our results are not particularly sensitive to this treatment of $M^{2}$. Indeed, the difference of the TFF results obtained with $M^{2}\left(Q^{2}\right)$ relative to those computed with a fixed $M^{2}$ value varies in the $Q^{2}$ range $[1 \div 40] \mathrm{GeV}^{2}$ from $-2 \%$ for $M^{2}=0.7 \mathrm{GeV}^{2}$ to $+3 \%$ to the maximum of $M^{2}=0.9 \mathrm{GeV}^{2}$. Would we set $M^{2}=1.1 \mathrm{GeV}^{2}$, a value well outside the interval mentioned above, the influence on the TFF in the relevant region of $Q^{2}$ between $10 \mathrm{GeV}^{2}$ and $40 \mathrm{GeV}^{2}$, in which our TFF predictions for the BMS "bunch" almost scale, would be not more than $4 \%$.

The leading-twist pion DA entering the LCSRs is determined in the framework of QCD SR NLCs 7]. Additional suppression results from the evolution of the Gegenbauer coefficients in the parameterization of the pion DA, taken into account in our analysis at the NLO level. The theoretical uncertainty entailed by the small photon virtuality accumulates as suppression expressed 
in the form of the narrow (red) strip below our original TFF predictions [4] in Fig. 2] This additional uncertainty somewhat increases at lower $Q^{2}$ values the width of the upper (green) band. The latter contains the variation of the shape of the pion DA in terms of $a_{2}, a_{4}$ within the framework of QCD SR NLCs [7] in conjunction with uncertainties owing to the twist-four coupling $\delta_{\mathrm{tw}-4}^{2}\left(\mu^{2}\right)=0.19 \pm 0.04 \mathrm{GeV}^{2}[22]$.

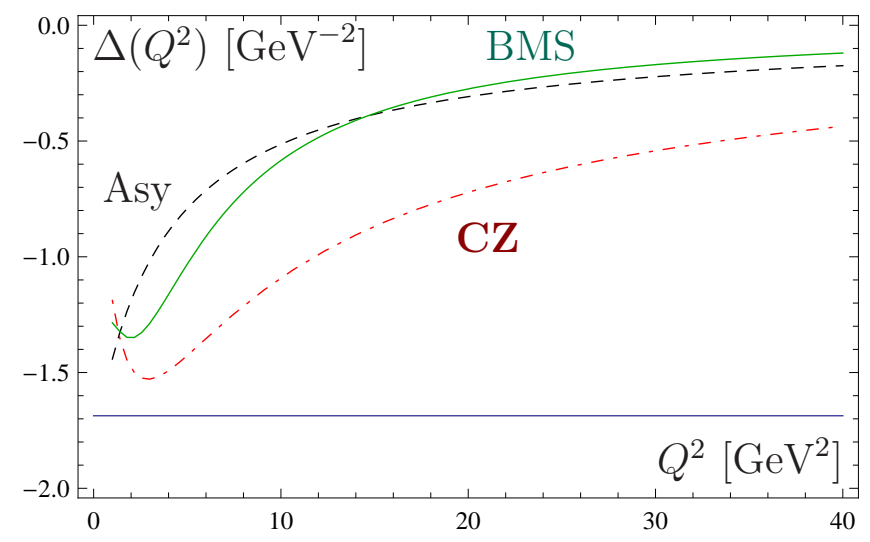

Figure 3: (color online). Parametrization of the dependence of the TFF on the small but finite photon virtuality defined in Eq. (17) in terms of $\Delta\left(Q^{2}\right)$ for three characteristic pion DAs: CZ 29] — dashed-dotted (red) line, BMS 7] — solid (green) line, Asy - dashed line. The solid horizontal line corresponds to the model in [8] that provides $\Delta=1 / m_{\rho}^{2}$.

To confront theory with single-tag experiments more precisely, we define the susceptibility (linear response)

$$
\Delta\left(Q^{2}\right) \equiv \frac{\left(\mathcal{F}^{\gamma \pi}\right)_{q^{2}=0}^{\prime}\left(Q^{2}\right)}{\mathcal{F}^{\gamma \pi}\left(Q^{2}\right)}
$$

which describes the relative sensitivity of the TFF to the variation of $q^{2}$. Thus, the relative change induced by a small virtuality of the quasi-real photon is $\delta \mathcal{F}^{\gamma \pi} / \mathcal{F}^{\gamma \pi}=$ $\Delta\left(Q^{2}\right) \cdot \delta q^{2}$, where the first factor represents the theoretical prediction, which contains the effects of strong interactions, and the second one is set by experiment. As a result, the TFF that includes the small-virtuality effect reads

$$
\tilde{\mathcal{F}}^{\gamma \pi}\left(Q^{2}, \delta q^{2}\right)=\mathcal{F}^{\gamma \pi}\left(Q^{2}\right)\left[1+\Delta\left(Q^{2}\right) \delta q^{2}\right] .
$$

Note that $\Delta\left(Q^{2}\right)$ depends on the shape of the pion DA employed in the calculation. This dependence is shown in Fig. 3 for the asymptotic (Asy) DA, and the BMS [7], and Chernyak-Zhitnitsky (CZ) [29] models. One observes from this figure that $\Delta\left(Q^{2}\right)$ has always a negative sign and provides suppression to $\tilde{\mathcal{F}}^{\gamma \pi}\left(Q^{2}, \delta q^{2}\right)$, starting at a maximum value of $-5.4 \%(-1.3 \%)$ at $Q^{2}=2 \mathrm{GeV}^{2}$ and rapidly decreasing to $-0.5 \%(-0.1 \%)$ for $Q^{2}>30 \mathrm{GeV}^{2}$, where we have used the values $\delta q^{2}=0.04(0.01) \mathrm{GeV}^{2}$ related to the Belle experiment [8]. The horizontal (blue) line in Fig. 3 shows an artificially reinforced constant response $\Delta$ obtained with a particular model used in [8].
Note that similar observations were done in Ref. 31] using Monte Carlo simulations in which a kinematic cut of $\left|q_{2}^{2}\right|<0.18 \mathrm{GeV}^{2}$ related to BABAR [1] was imposed and found to lead to a reduction of the cross section at the level of $3 \%$ for the whole $Q^{2}$ range from 1 to $35 \mathrm{GeV}^{2}$.

The main conclusions from these findings are twofold: First, a more precise comparison of theoretical predictions with the experimental data should account for the final virtuality of the quasi-real photon because it induces a non-negligible effect. Second, any calculation with a QCD-based $\pi$ DA model will receive additional suppression so that the chances to reconcile theoretical predictions with the $B A B A R$ data will decrease even further, with the asymptotic DA loosing ground against all existing data. In contrast, the enlarged band of our theoretical predictions, obtained from the BMS "bunch" of $\pi$ DAs (Fig. 2) still includes all CLEO data with their error bars and most of the Belle data, while even the BABAR data below $10 \mathrm{GeV}^{2}$ are also covered.

\section{B. 3D pion DA models from NLC QCD SRs}

As mentioned above, the "BMS" bunch of pion DAs is based on the first two (nontrivial) Gegenbauer harmonics. The corresponding coefficients $a_{2}$ and $a_{4}$ were derived from the first five $\left\langle\xi^{2 n}\right\rangle$ moments, estimated in [7, 32 using QCD SRs with NLCs. It was found that the next coefficients $a_{6}, a_{8}, a_{10}$, obtained this way, can be set equal to zero, though they bear rather large uncertainties. On the other hand, the pion DAs constructed with the minimal subset $\left(a_{2}, a_{4}\right)$ provide a sufficiently good description of different pion observables - see [26] for a review. Motivated by the high- $Q^{2}$ Belle data that are not adequately described with only two Gegenbauer coefficients [13], we include into our analysis of the data the next higher term $a_{6} \approx 0.038 \sim 0$ with its associated uncertainties ranging within $[-0.186 \div 0.263]$ in the sense of correlated "noise", but disregard still higher terms. The outcome of this procedure is displayed in Fig. 4 in which the inclusion of $a_{6}$ with increasing values is illustrated as a 3D flight of "stairs" of slanted rectangles.

The axis of this "stairs" incidentally crosses the center of the CCBe ellipsoid (larger ellipsoid in the forefront). One observes that there is no way to satisfy the theoretical constraints ("stairs" of slanted rectangles) and the CCBB set of the data (smaller ellipsoid in the background).

The predictions for the TFF obtained with the threeparametric pion DA "bunch" are shown in Fig. 5 by means of a broader (blue) band enveloping the original (green) one. One appreciates that the width of the (blue) enveloping band becomes larger above $Q^{2} \approx 10 \mathrm{GeV}^{2}$, while its deviation from the original (green) strip below that scale is marginal. The bottom line is that the DAs from our new 3D "bunch" achieve a rather good agreement with the CCBe data at the expense of larger error bars of the calculated TFF, while even this enlarged band 
of predictions is conflicting with the $B A B A R$ data above $Q^{2} \simeq 10 \mathrm{GeV}^{2}$.

\section{STATISTICAL DATA ANALYSIS}

Before we continue with the statistical analysis of the real data, it is advisable to state what we should expect from the point of view of QCD. If we believe that QCD is the correct microscopic theory of strong interactions, then it is reasonable to suppose that the data would cluster with increasing $Q^{2}$ more and more closely around the limiting value $\sqrt{2} f_{\pi}$ becoming equal to that value (within error bars), if a fictitious experiment were continued to remote momentum scales.

The antithetic trend between the BABAR [1] and the Belle [8] data for the $\gamma^{*} \gamma \pi^{0}$ TFF calls for a careful statistical evaluation. The crucial question is whether these

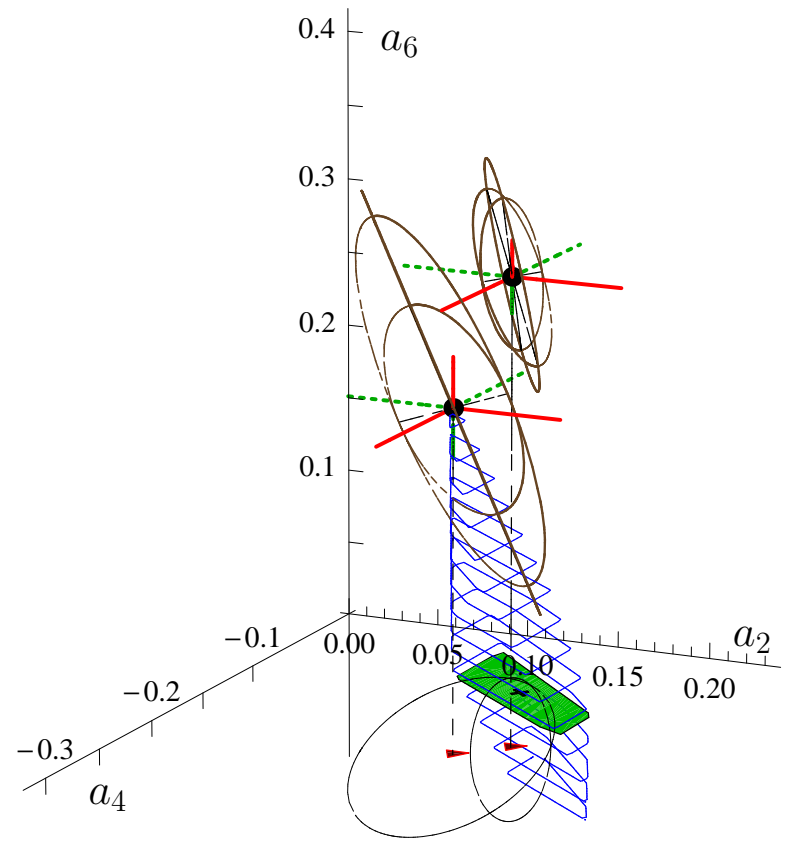

Figure 4: (color online). 3D graphics of the pion DA "bunch" obtained from QCD SRs with NLCs, in terms of the coefficients $a_{2}, a_{4}, a_{6}$, shown as a flight of "stairs" of slanted rectangles, while the original BMS "bunch" in the plane $\left(a_{2}, a_{4}\right)$ is shown as a (green) rectangle. The displayed $1 \sigma$-error ellipsoids represent fits to two data sets: smaller ellipsoid (CCBB) [1, 9, 10] and larger ellipsoid (CCBe) [8 10]. The theoretical $\Delta \delta_{\mathrm{tw} 4}^{2}$ errors for an increasing value of $\delta_{\mathrm{tw} 4}^{2}$ are indicated by a solid (red) hairline cross in the forefront, whereas a dashed (green) hairline cross in the background denotes a decreasing value. All displayed results were calculated at the scale $\mu=2.4 \mathrm{GeV}$, with more explanations being given in Sec. IIIB.

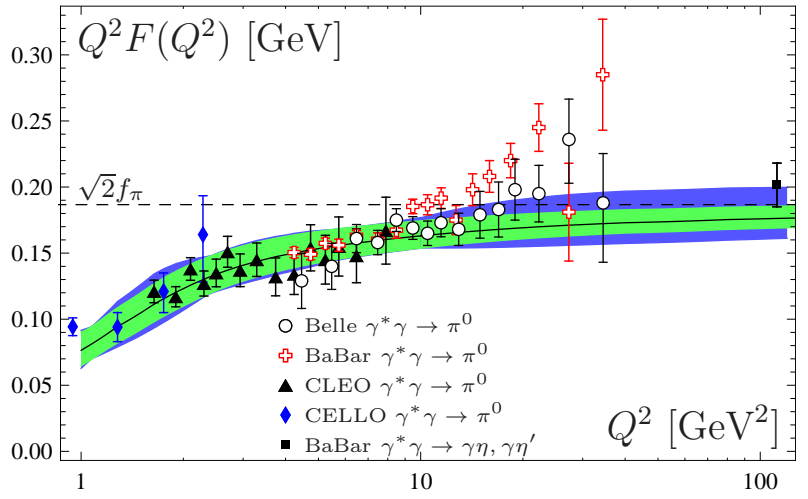

Figure 5: (color online). The broader (blue) band, enclosing the narrower (green) one, displays the theoretical predictions for the scaled $\gamma^{*} \gamma \pi^{0}$ TFF obtained with the LCSR approach and pion DAs extracted from the $\left\langle\xi^{n}\right\rangle$-moments from 7] using three Gegenbauer coefficients $a_{2}, a_{4}, a_{6}$. The narrower (green) strip reproduces the results of the original "BMS bunch", which is shown in Fig. 4 as a shaded slanted rectangle in terms of $a_{2}$ and $a_{4}$. For comparison, experimental data from various collaborations with the indicated labels are also shown.

data sets are mutually supportive or exclusive. In other words, can we predict the trend of the Belle data using as learning input the BABAR data and vice versa? To answer this question, we use two different parameterizations: a dipole fit [8, 10]

$$
Q^{2}\left|F\left(Q^{2}\right)\right|=\frac{B Q^{2}}{Q^{2}+C}
$$

and a power-law fit [1, [8]

$$
Q^{2}\left|F\left(Q^{2}\right)\right|=A\left(\frac{Q^{2}}{10 \mathrm{GeV}^{2}}\right)^{\beta},
$$

where $B, C, A, \beta$ are free fit parameters. Both types of fitting functions have been used by experimentalists 1, 8, 10] before because of their convenience. However, we could equally well use another fitting function - it doesn't really matter.

What matters most is the mutual consistency of such fits in predicting the trend of the data one from the other. We use the following convenient abbreviations: $B A B A R \equiv \mathfrak{B}$, Belle $\equiv \mathfrak{b}$, Dipole $\equiv \mathrm{D}$, Power-law $\equiv \mathrm{P}$ and express the goodness of fit for each parametrization in terms of $\chi_{\text {ndf }}^{2}$ (data set, fit model). We employ the two mathematical expressions given above to determine the fit parameters $B, C$ and $A, \beta$ using in turn as input the $B A B A R(\mathfrak{B})$ and the Belle $(\mathfrak{b})$ data. Then, we test how good the obtained fitting model can describe the other set of data. The results of this data processing are given in Table I. For future use with respect to fits to the other data sets CLEO and CELLO, abbreviated in common by $\mathfrak{C} \mathfrak{C}$, we define a relative goodness of fit criterion $\chi_{\text {ndf }}^{2}$ (data set- $1 \rightarrow$ data set-2, fit model) that serves to explore how the various sets compare to each 
Table I: Results of the statistical analysis of the BABAR (abbreviated by $\mathfrak{B}$ ) [1], the Belle [8] (abbreviated by $\mathfrak{b}$ ), and CELLO\&CLEO data 9, 10] (abbreviated by $\mathfrak{C} \mathfrak{C}$ ) in terms of a dipole fit (denoted D) and a power-law fit (denoted P), gauging the accuracy by a goodness of fit $\chi_{\text {ndf }}^{2}$ and the units of the corresponding $\sigma$, as described in the text.

\begin{tabular}{llllccc}
\hline \hline Symbol/Name & Fit & \multicolumn{2}{c}{ Best fit values } & $\chi^{2}$ & Relative $\chi^{2}$ & $\sigma$ deviation \\
\hline $\mathfrak{A} / \mathfrak{B}$ & $\mathrm{D}$ & $B_{\mathfrak{B}}=0.23$ & $C_{\mathfrak{B}}=2.6$ & $\chi^{2}(\mathfrak{B}, \mathrm{D})=1.7$ & $\chi^{2}(\mathfrak{B} \rightarrow \mathfrak{b}, \mathrm{D})=1.5$ & $\Delta_{\mathfrak{B} \rightarrow \mathfrak{b}, \mathrm{D}}=7.2$ \\
$\mathfrak{B}$ & $\mathrm{P}$ & $A_{\mathfrak{B}}=0.182$ & $\beta_{\mathfrak{B}}=0.25$ & $\chi^{2}(\mathfrak{B}, \mathrm{P})=1.0$ & $\chi^{2}(\mathfrak{B} \rightarrow \mathfrak{b}, \mathrm{P})=1.7$ & $\Delta_{\mathfrak{B} \rightarrow \mathfrak{b}, \mathrm{P}}=6.0$ \\
\hline $\mathrm{O} / \mathfrak{b}$ & $\mathrm{D}$ & $B_{\mathfrak{b}}=0.212$ & $C_{\mathfrak{b}}=2.4$ & $\chi^{2}(\mathfrak{b}, \mathrm{D})=0.4$ & $\chi^{2}(\mathfrak{b} \rightarrow \mathfrak{B}, \mathrm{D})=5.5$ & $\Delta_{\mathfrak{b} \rightarrow \mathfrak{B}, \mathrm{D}}=3.3$ \\
$\mathfrak{b}$ & $\mathrm{P}$ & $A_{\mathfrak{b}}=0.169$ & $\beta_{\mathfrak{b}}=0.19$ & $\chi^{2}(\mathfrak{b}, \mathrm{P})=0.4$ & $\chi^{2}(\mathfrak{b} \rightarrow \mathfrak{B}, \mathrm{P})=3.7$ & $\Delta_{\mathfrak{b} \rightarrow \mathfrak{B}, \mathrm{P}}=3.7$ \\
\hline \hline $\boldsymbol{\Lambda} / \mathfrak{C C}$ & $\mathrm{D}$ & $B_{\mathfrak{C} \mathfrak{C}}=0.176$ & $C_{\mathfrak{B}}=0.82$ & $\chi^{2}(\mathfrak{C C}, \mathrm{D})=0.6$ & $\chi^{2}(\mathfrak{C C} \rightarrow \mathfrak{b}, \mathrm{D})=1.0$ & $\Delta_{\mathfrak{C} \mathfrak{C} \rightarrow \mathfrak{b}, \mathrm{D}}=8.7$ \\
$\mathbf{0} / \mathrm{BL}$ & $\mathrm{D}$ & $B_{\mathrm{BL}}=0.187$ & $C_{\mathrm{BL}}=0.69$ & - & - & - \\
$\boldsymbol{\nabla} / \mathrm{LCSR}$ & $\mathrm{D}$ & $B_{\mathrm{LCSR}}=0.180$ & $C_{\mathrm{LCSR}}=1.00$ & - & - & - \\
\hline \hline
\end{tabular}

other., i.e., how well the best fit (fit model with bestfit parameters), obtained from the learning data (set-1), can predict the test data (set-2). As an example we note $\chi_{\text {ndf }}^{2}(\mathfrak{B} \rightarrow \mathfrak{b}, D)$ which describes the fitting of the Belle data from those of BABAR using the dipole formula.

The strong variation of $\chi_{\text {ndf }}^{2}$ in the upper part of Table I reveals that the dynamical behavior with $Q^{2}$ of the real Belle data cannot be accurately predicted on the basis of the BABAR data neither with the dipole form nor with the power-law one. Indeed the $\chi_{\text {ndf }}^{2}$ values $\chi_{\text {ndf }}^{2}(\mathfrak{B} \rightarrow \mathfrak{b}, D)=1.5$ (dipole fit) and $\chi_{\text {ndf }}^{2}(\mathfrak{B} \rightarrow$ $\mathfrak{b}, \mathrm{P})=1.8$ (power-law fit) obtained from the coefficients $(B, C)$ and $(A, \beta)$ via the $B A B A R$ data are much larger relative to the value $\chi_{\text {ndf }}^{2}(\mathfrak{b}, \mathrm{D})=\chi_{\text {ndf }}^{2}(\mathfrak{b}, \mathrm{P})=0.4$, determined directly from the Belle data. But also the inverse prediction has not an acceptable precision. Using the values of $(B, C)$, determined from the Belle data, we find that the $B A B A R$ data can be fitted by the dipole fit with a $\chi_{\text {ndf }}^{2}(\mathfrak{b} \rightarrow \mathfrak{B}, \mathrm{D})=5.4$, while the analogous coefficients $(A, \beta)$ of the power-law fit would give $\chi_{\text {ndf }}^{2}(\mathfrak{b} \rightarrow \mathfrak{B}, \mathrm{P})=3.7$.

What remains contentious is whether one should prune the outliers in both data sets. BABAR and Belle made no attempt to explain the origin of the corresponding outliers, but simply accepted them as a given feature of their data representing the tails of their probability distribution. Removing the two BABAR outliers, would entail a slightly worse description of the Belle data. On the other hand, pruning the single outlier of the Belle data would further improve the scaling behavior of the data with $Q^{2}$ and increase the tension to the BABAR data. In both cases, removing the big leaps that are underestimated by the typical gaussian distribution of each data set, the differences between successive values of the TFF (called in statistics the "returns") would remain above $10 \mathrm{GeV}^{2}$ practically steady and close to zero, revealing the underlying scaling behavior of the Belle TFF data, while emphasizing at the same time the auxetic trend of the $B A B A R$ data in this $Q^{2}$ region.

Another important observation from Table \is that the particular form of the parametrization used to fit the data is not crucial. In fact, the Belle data [8] can be described with both functional forms - dipole and power-law - with exactly the same accuracy: $\chi_{\text {ndf }}^{2}(b, D)=\chi_{\text {ndf }}^{2}(b, P)=$ 0.4. Using another parametrization would not change these findings significantly. Thus, without any theoretical presumption, the statistical analysis of the Belle and the BABAR data suggests that they segregate into two distinct classes of data and cannot merge into a single pool of aggregated data. This finding reinforces our conclusions drawn in [11] that one should divide the data into two discrete classes with reference to their $Q^{2}$ behavior: one showing scaling (Belle data) and the other exhibiting auxesis (BABAR data).

The above discussion can be given a more quantitative meaning by displaying the precise statistical information linked to each of the above fits by means of Fig. 6. The left panel shows the dipole fit to the CELLO, CLEO, $B A B A R$, and Belle data in terms of the parameters $B$ and $C$, cf. Eq. (9), while the right panel contains an analogous graphics for a power-law fit in terms of the parameters $A$ and $\beta$ (see Eq. (10)). We display the $1 \sigma$ error ellipses, associated with the indicated data sets, and mark their centers by a flag which shows the following values from top to bottom. First flag: The first number is $\chi_{\text {ndf }}^{2}$ for this data set and the second one its $\sigma$ value. Second flag: $\chi_{\text {ndf }}^{2}$ (marked data set $\rightarrow$ this data set) and $\sigma$ with respect to the marked center as seen from the data set in the first flag; third flag: in analogy to the previous one but with another marked center. The marks for the ellipse centers are displayed in the figure and are also listed here for convenience: Cello and CLEO data: $\boldsymbol{\Delta}$; Belle data: $\bigcirc$; BABAR data: ↔. The point labeled by corresponds to the Brodsky-Lepage (BL) interpolation formula (11) given in the next Section, while the symbol $\nabla$ corresponds to the result of the LCSR calculation which employs the BMS DA model. 

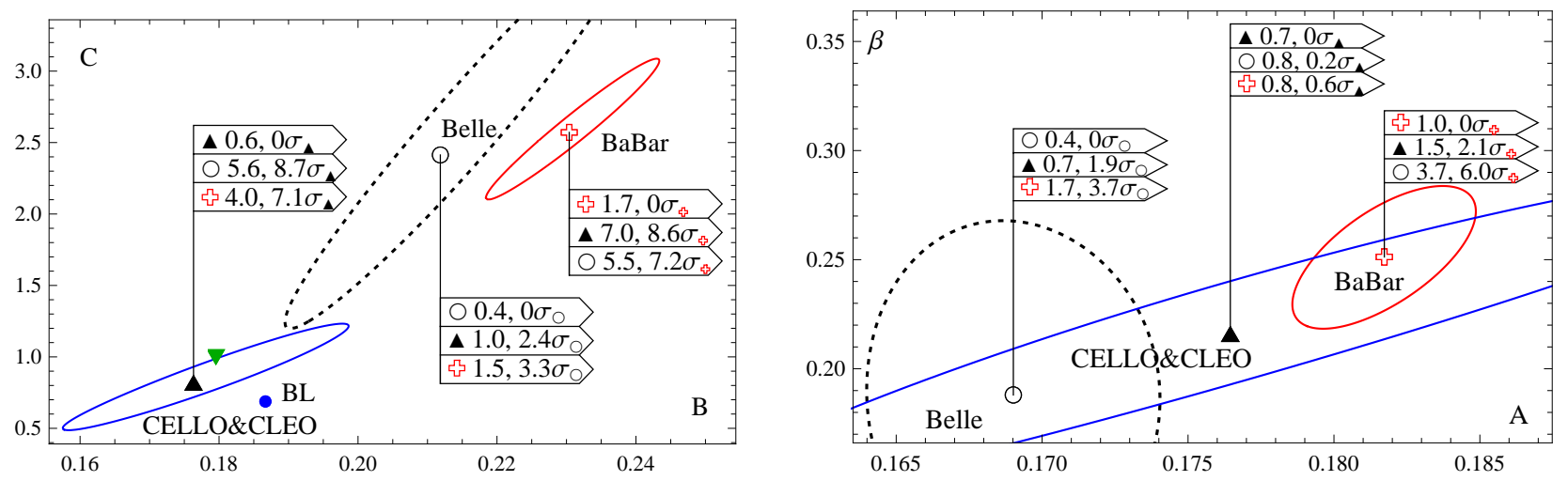

Figure 6: Left. Dipole fit to the CELLO, CLEO, BABAR, and Belle data, described in terms of the parameters $B$ and $C$, cf. Eq. (9). Right. Analogous graphics for the power-law fit in terms of the parameters $A$ and $\beta$, cf. Eq. (10). More explanations are given in the text.

\section{DISCUSSION OF RESULTS}

In the previous section we concentrated on the statistical analysis of the experimental data without attempting to provide deeper explanations. Here we turn to a discussion of the nature and the causes of the antithetic trends of the present data from the point of view of theory.

There are two rigorous predictions for the behavior of the $\pi^{0}$ TFF, one extracted form the axial anomaly in the chiral limit of QCD, i.e., at $Q^{2}=0$ 33, 34, and the other obtained from QCD in the asymptotic limit $Q^{2} \rightarrow \infty$ 35]. To interpolate between the $Q^{2}=0$ and $Q^{2}=\infty$ limits, the following phenomenological monopole form has been proposed Brodsky and Lepage (BL) in [35]:

$$
F^{\gamma^{*} \gamma \pi}\left(Q^{2}\right)=\frac{\sqrt{2} f_{\pi}}{4 \pi^{2} f_{\pi}^{2}+Q^{2}} .
$$

One can derive analogous interpolation formulas for the other pseudoscalar mesons with $J^{\mathrm{PC}}=0^{-+}$, i.e., the $\eta$ and $\eta^{\prime}$ in terms of their decay constants $f_{\eta}$ and $f_{\eta^{\prime}}$.

Though we lack a detailed theoretical scheme to deal precisely with nonperturbative QCD, we would expect that the experimental data would comply with the above QCD preconceptions of the $\pi^{0} \mathrm{TFF}$. While this is true for the CLEO and most of the Belle data, the BABAR data indicate a different trend at momenta $Q^{2} \gtrsim 10 \mathrm{GeV}^{2}$ that is characterized by a distinctive increase. Hence, from the QCD point of view, these data appear as being contingent on unknown enhancement mechanisms of the nonperturbative quark-gluon interactions. While we understand the mechanism of endpoint suppressionnonlocal quark/gluon condensates 7] —we have no clear understanding of the mechanism of endpoint enhancement which would give rise to a flat-top pion DA and lead to an auxetic behavior of the pion-photon transition form factor. A flat-top pion DA was proposed by Radyushkin [20] and in a different context also by Polyakov 21], while hints for a flat-like pion DA were obtained earlier within the Nambu-Jona-Lasinio model [36] and also in the so-called Spectral Quark Model [37], as well as from the instanton vacuum [38, 39]. Such DAs entail a logarithmic rise of $\mathcal{F}^{\gamma \pi}\left(Q^{2}\right)$ and can indeed comply with the trend of the $B A B A R$ data for the $\pi \gamma$ transition. (see [40, 41]). Meanwhile, several authors have proposed contextual explanations in conjunction with particular low-energy models that can indeed replicate the growing behavior of $\mathcal{F}^{\gamma \pi}\left(Q^{2}\right)$ indicated by the BABAR data. Besides the analyses already mentioned, examples are given by the works in Refs. [42 51]. However, strictly speaking, the flat-top pion DA is an after-the-fact rationalization of the rising scaled TFF without support from the standard QCD framework. Thus, it is of little consolation to appeal to contextual explanations of this effect, though it is possible that some deeper reason for enhancement may exist - see, for instance, [21, 41, 52, 53].

The statistical analysis of the data in this work has shown that the auxetic trend of the BABAR data above $10 \mathrm{GeV}^{2}$ cannot be predicted from other experimental data antecedent to them [9, 10]. Also the new Belle data [8] cannot be used to retrospectively "predict" such a behavior of the $\pi \gamma$ TFF using a popular parametrization like the dipole or the power-law fit (as we have shown in the previous section). From the theoretical side, the rise of the TFF cannot be intuited within the standard framework of QCD based on collinear factorization as well. Therefore, it is a futile endeavor to try to explain the auxetic behavior of the BABAR data by systematically engineering the fit to these data as long as there is no deeper understanding of some underlying dynamical mechanism that should reveal itself also in other QCD processes.

Comparing our theoretical predictions, computed with the method of LCSRs and using as nonperturbative input the $\pi$ DAs extracted from QCD SRs with NLCs, we argue that they provide reasonable agreement with almost all data - except those of $B A B A R$ beyond $10 \mathrm{GeV}^{2}$ (see Fig. (7). Viewed as a function of $Q^{2}$, the calculated TFF has two parts in succession: a gentle ascent up to about $10 \mathrm{GeV}^{2}$, followed by a saturated part exhibiting scaling 


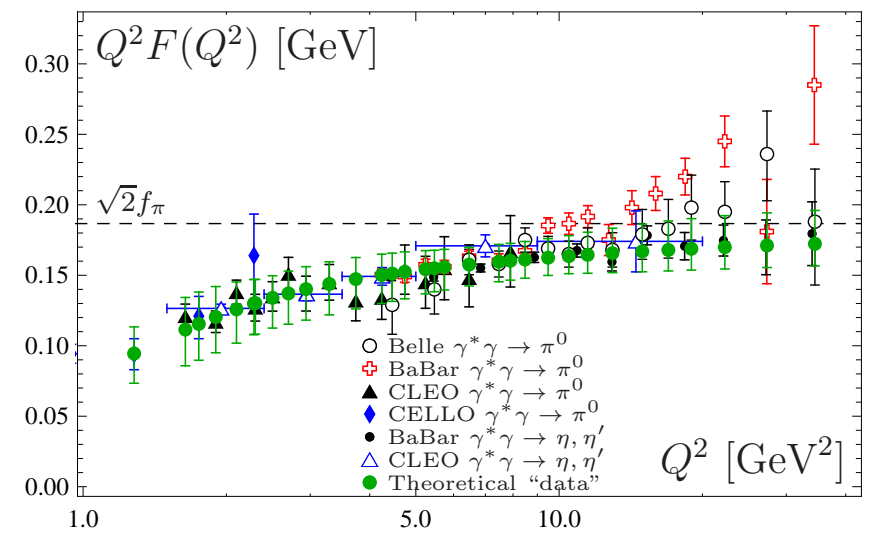

Figure 7: (color online). Theoretical predictions for the scaled $\gamma^{*} \gamma \pi^{0}$ transition form factor in the form of "theoretical data", including all uncertainties considered in the text, in comparison with real experimental data taken from various experiments with designations as indicated. A logarithmic scale for $Q^{2}$ is used.

beyond that scale in accordance with perturbative QCD. Indeed, as one observes from this figure, our predictions shown in the form of "theoretical data" (green bullets with error bars) comply pretty well with the Belle data within the estimated uncertainty range, but disagree with the high- $Q^{2}$ tail of the BABAR data. At the expense of accepting some "noise" for the coefficient $a_{6}$, the enlarged error bars of our predictions increase the agreement with the Belle data significantly, while no reconciliation with the high $Q^{2}$ tail of the BABAR data is achieved. This incongruity is divisive in a broader sense because it tells us that the BABAR data are incompatible with scaling of the TFF at large $Q^{2}$, a behavior that is a basic characteristic of any QCD-based calculation. On the other hand, the high- $Q^{2}$ trend of the Belle data supports the scaling behavior of the TFF. These opposing tendencies cannot be reconciled until more data will become available in the future.

Abstracting from the $\pi^{0} B A B A R$ data, an antagonistic mechanism, governed by quark-gluon strong interactions, that can provide such a distinctive enhancement to the TFF at large $Q^{2}$ has yet to be identified. One may think that this could eventually be the result of multiple correlations with various correlation lengths, related to constructive interference effects, that may prevent partonic interactions governed by fixed-order or resummed QCD perturbation theory up to excessively large momentum transfers. As we have recently argued in [1], the antithetic trends of the BABAR and the Belle data, pertaining invariably to auxesis vs. scaling, correspond to $\pi$ DAs with distinct endpoint characteristics. To get a scaling behavior, one needs endpoint suppression but also a shape that is wider than the asymptotic DA. As we have shown in this analysis, the asymptotic DA falls short to comply with all existing data. On the other hand, endpoint enhancement with only one but excessively large coefficient $a_{2}$, like in the case of the CZ DA, overestimates all data below $20 \mathrm{GeV}^{2}$ while being unable at the same time to reproduce the (at least) logarithmic increase of the high- $Q^{2} B A B A R$ data. This is only possible if one includes into the $\pi^{0} \mathrm{DA}$ more coefficients, as proposed in [6, 24]. Employing a flat-top $\pi$ DA [20, 21], the agreement with the BABAR data is best but at the expense that one has to abandon collinear factorization and QCD scaling.

As we discussed in more detail in [4], our approach is capable of capturing the basic features of the $\gamma^{*} \gamma \eta\left(\eta^{\prime}\right)$ TFF as well. Using for simplicity the description of the $\eta-\eta^{\prime}$ mixing in the quark-flavor basis [19] (see [54] for a review), one has

$$
\left(\begin{array}{c}
|\eta\rangle \\
\left|\eta^{\prime}\right\rangle
\end{array}\right)=\left(\begin{array}{cc}
\cos \phi & -\sin \phi \\
\sin \phi & \cos \phi
\end{array}\right)\left(\begin{array}{l}
|n\rangle \\
|S\rangle
\end{array}\right),
$$

where the nonstrange part is given by $|n\rangle=$ $(1 / \sqrt{2})(|u \bar{u}\rangle+|d \bar{d}\rangle)$ and the strange component is $|S\rangle=$ $|s \bar{s}\rangle$, with the angle $\phi$ denoting the deviation of the mixing angle from the ideal one owing to the $U_{\mathrm{A}}(1)$, i.e., the axial-vector, anomaly. Then, the TFFs of the physical $\eta$ and $\eta^{\prime}$ mesons can be linked to those of the states $|n\rangle$ and $|S\rangle$ - see [18] for further details. Using the currently accepted value of the mixing angle $\phi \approx 41^{\circ}$, as used by the BABAR Collaboration in [18], in order to mix the data on $\eta$ and $\eta^{\prime}$, we obtain the data points for the TFF of the $|n\rangle$ state displayed in Fig. 7. This rough treatment ignores in the $Q^{2}$ evolution the mixing with the gluonic components and also the difference in the normalization owing to the different decay constants, but is sufficient for our qualitative considerations.

An independent confirmation of the $\gamma^{*} \gamma \eta\left(\eta^{\prime}\right)$ [18] data, would establish the agreement with the asymptotic QCD limit, denoted by the horizontal dashed line in Fig. 7 , It would also agree with our theoretical predictions obtained with the BMS formalism ("theoretical" data in the same figure), as we explained above. This would mean that (a) the DAs of the two pseudoscalar mesons $\pi^{0}$ and $\eta$ (strictly speaking its nonstrange component) are similar - no (significant) $S U(3)_{\mathrm{F}}$ flavor asymmetry - and (b) have their endpoints strongly suppressed. Implicitly, this would give support to the idea of NLCs that entail this suppression and thus validate the sum-rule method in 7] based on them. In contrast, the analysis in [55] claims good agreement with the BABAR data for the $\eta$ TFF using an endpoint-enhanced $\eta$ DA derived from the Nambu-Jona-Lasinio model. A scheme to describe the $\eta-\eta^{\prime}$ mixing with two decay parameters $\left(f_{0}\right.$ and $\left.f_{8}\right)$ and two mixing angles $\left(\theta_{0}\right.$ and $\left.\theta_{8}\right)$ was recently used in [31]. These authors find that their TFF calculation for the two-octet ansatz is consistent with the bulk of the available data. At the same time, the $\pi^{0} \mathrm{TFF}$ disagrees with the BABAR data for both the one-octet and the two-octet ansatz. A more dedicated analysis of the TFFs of the $\eta-\eta^{\prime}$ system and their mixing properties is given in [56, 57. 


\section{CONCLUSIONS}

We presented an analysis that illustrates and discusses the complex spectrum of challenges encountered in the statistical evaluation of the various experimental data on the pion-photon TFF and their interpretation. Using two different common models (dipole and power-law) to fit the CELLO, CLEO, BABAR, and Belle data, we showed that it is not possible to predict their trends one from another with acceptable accuracy. In particular the rapid growth of the high- $Q^{2} B A B A R$ data cannot be retrospectively predicted from the Belle data. On the other hand, such an auxetic behavior of the TFF as indicated by the $B A B A R$ data can hardly be reconciled with the trend of the Belle data that is compatible with scaling, irrespective of the fit model used. In fact, both fit models describe the Belle data with almost the same statistical precision (Table I).

From the theoretical side, we studied in this work the effect of a non-vanishing small virtuality of the quasi-real photon on the TFF within the LCSR framework. Though the ensuing suppression of the TFF is rather small for the values associated with the Belle experiment, this effect is not negligible - especially at lower and moderate $Q^{2}$ values - and makes it clear that the asymptotic pion DA cannot be considered as a serious candidate for the description of the data. In this context let us remark that our predictions in the intermediate $Q^{2}$ domain (28) $\mathrm{GeV}^{2}$ also agree in trend with feasibility studies of the BES-III Collaboration [58] based on a fit to the transition form factor data of the BABAR Collaboration 11. Moreover, the expected high accuracy of this measurement below $5 \mathrm{GeV}^{2} 71$ will provide a means of excluding pion DAs that yield a scaled TFF with a steep increase just in this region. The high accuracy of the data may also help reducing the uncertainty in the extraction of the vacuum quark virtuality $\lambda_{q}^{2}$ that controls the shape of the twist-two pion DA and also the strength of the twist-four coupling $\delta_{\mathrm{tw}-4}^{2} \approx \lambda_{q}^{2} / 2$, see Appendix $\mathrm{A}$ in [22].

A second theoretical ingredient of our investigation is the inclusion of the Gegenbauer coefficient $a_{6}$ into the theoretical scheme to calculate the pion DA and the pionphoton TFF, considering its uncertainties as correlated "noise". While a finite photon virtuality influences the TFF predictions at lower values of $Q^{2}$ causing suppression, the inclusion of a third parameter in the representation of the pion DA affects the result for the TFF mainly in the large- $Q^{2}$ domain by increasing the error width of the theoretical band of predictions. This suffices to increase even further the compliance of our predictions with the Belle data (Fig. 5 ).

Indeed, our current investigation in conjunction with our recent works [4, 11, 13] gives evidence that, staying within the standard QCD approach based on collinear factorization, the best overall agreement with all available data (cf. Fig. (7) is provided by BMS-like pion DAs that represent a compromise between two conflicting urges: to have enough enhancement in the lower $Q^{2}$ in order to reach the data from below, while, on the other hand, to limit that enhancement from above at higher $Q^{2}$ so that the scaled TFF saturates and scaling prevails. Clearly, such a behavior cannot comply with a powerlaw which is an indication that the system is scale-free. In fact, an auxetic TFF would correspond to a flat-top pion DA whose main characteristic is that there are no features at some value of $x$ that makes that particular longitudinal-momentum fraction stand out. There are no "dips" and "humps" anywhere. This scaling behavior with $x$ lends itself to a pion interpretation as being a "pointlike" particle [59], in the sense that it behaves as a unit without revealing its internal constituents, despite the large momentum $Q^{2}$ with which it was probed.

It is worth mentioning that the predictions for the pion TFF extracted from two independent AdS/QCD approaches [60, 61] (see also 62]) disagree with the high- $Q^{2}$ trend of the BABAR data, while being in good agreement with the results of the BMS formalism. A full scale explanation of the antithetic trend of the BABAR data relative to Belle and to the BABAR data on the $\eta\left(\eta^{\prime}\right)-\gamma$ transition within the confines of QCD cannot be given at present. New experimental data on the spacelike TFFs of the pseudoscalar mesons $\pi^{0}, \eta, \eta^{\prime}$ could provide a litmus test of the corresponding DAs of these hadrons shedding also light on the underlying mechanisms of QCD to create them.

\section{Acknowledgments}

A.V.P. is thankful to Prof. Vicente Vento and Prof. Pedro Gonzalez for the warm hospitality at the University of Valencia, where part of this investigation was carried out. The work of A.V.P. was supported in part by the Ministry of Education and Science of the Russian Federation, project 14.B37.21.0910, and by HadronPhysics2, Spanish Ministerio de Economia y Competitividad and EU FEDER under contract FPA2010-21750C02-01, AIC10-D-000598, and GVPrometeo2009/129. We acknowledge support from the Heisenberg-Landau Program under Grants 2012 and 2013, the Russian Foundation for Fundamental Research (Grant No. 12-0200613a, 11-01-00182a).

\section{Appendix A: Form Factors}

We have worked out in Eq. (41) the transition form factor and its first derivative with respect to $q^{2}$. Their explicit forms are

$$
\begin{aligned}
& \mathcal{F}^{\gamma \pi}\left(Q^{2}\right)=\frac{\sqrt{2}}{3} f_{\pi}\left[\frac{Q^{2}}{m_{\rho}^{2}} \int_{x_{0}}^{1} \exp \left(\frac{m_{\rho}^{2}-Q^{2} \bar{x} / x}{M^{2}}\right)\right. \\
& \left.\quad \times \bar{\rho}\left(Q^{2}, x\right) \frac{d x}{x}+\int_{0}^{x_{0}} \bar{\rho}\left(Q^{2}, x\right) \frac{d x}{\bar{x}}\right]
\end{aligned}
$$




$$
\begin{aligned}
-\left(\mathcal{F}^{\gamma \pi}\right)_{q^{2}=0}^{\prime} & \left(Q^{2}\right)=\frac{\sqrt{2}}{3} f_{\pi}\left[\frac{Q^{2}}{m_{\rho}^{4}} \int_{x_{0}}^{1} \exp \left(\frac{m_{\rho}^{2}-Q^{2} \bar{x} / x}{M^{2}}\right)\right. \\
& \left.\times \bar{\rho}\left(Q^{2}, x\right) \frac{d x}{x}+\int_{0}^{x_{0}} \frac{\bar{\rho}\left(Q^{2}, x\right)}{Q^{2}} \frac{x}{\bar{x}^{2}} d x\right] \cdot \quad \text { (A2) }
\end{aligned}
$$

With the above expressions one can compute the susceptibility ("linear response") $\Delta\left(Q^{2}\right)$ defined in Eq. (7). The common spectral density $\bar{\rho}$ for the sum of the twisttwo and twist-four contributions is

$$
\begin{aligned}
\bar{\rho}\left(Q^{2}, x\right)=\sum_{n=0} a_{n}\left(Q^{2}, \mu^{2}\right) \bar{\rho}_{n}\left(Q^{2}, x\right) \\
+\frac{\delta_{\mathrm{tw}-4}^{2}}{Q^{2}} x \frac{d}{d x} \varphi^{(4)}(x), \\
\bar{\rho}_{n}\left(Q^{2}, x\right)=\bar{\rho}_{n}^{(0)}(x)+a_{s}^{1} \bar{\rho}_{n}^{(1)}\left(Q^{2}, x\right) \\
+a_{s}^{2} \bar{\rho}_{n}^{(2)}\left(Q^{2}, x\right)+\ldots
\end{aligned}
$$

The expansion on the l.h.s. of A3 corresponds to the expansion of the twist-two pion DA $\varphi_{\pi}$ over the set of the Gegenbauer polynomials, $\psi_{n}(x)=6 x \bar{x} C_{n}^{(3 / 2)}(x-\bar{x})$, $\varphi\left(x, \mu^{2}\right)=\sum_{n=0} a_{n}\left(\mu^{2}\right) \psi_{n}(x)$, with $v(n), v^{b}(n)$ being the eigenvalues of the LO Efremov-Radyushkin-BrodskyLepage (ERBL) equations 63 65], whereas $G_{n l}$ and $b_{n l}$ are calculable triangular matrices calculated for the first time in [2] and corrected later in [6] (see there for more details). The relevant expressions read

$$
\begin{aligned}
& \bar{\rho}_{n}^{(0)}(x)=\psi_{n}(x) ; \quad \varphi^{(4)}(x)=\frac{80}{3} x^{2}(1-x)^{2}, \\
& \bar{\rho}_{n}^{(1)}\left(Q^{2}, \mu_{\mathrm{F}}^{2} ; x\right) \frac{1}{C_{\mathrm{F}}}=\left\{-3\left[1+v^{b}(n)\right]+\frac{\pi^{2}}{3}+2 v(n) \ln \left(\frac{Q^{2}}{\mu_{\mathrm{F}}^{2}}\right)\right\} \psi_{n}(x) \\
& +\left\{2 v(n) \ln \left(\frac{\bar{x}}{x}\right)-\ln ^{2}\left(\frac{\bar{x}}{x}\right)\right\} \psi_{n}(x)-2\left[\sum_{l=0,2, \ldots}^{n} G_{n l} \psi_{l}(x)+v(n)\left(\sum_{l=0,1, \ldots}^{n} b_{n l} \psi_{l}(x)-3 \bar{x}\right)\right] .
\end{aligned}
$$

\section{Appendix B: Hard part of the TFF}

To obtain the contributions to the "hard part" of the TFF, $\int_{0}^{x} \bar{\rho}_{n}\left(Q^{2}, t\right) \frac{1}{(\bar{t})} d t$, Eq. (A1), and those to the corresponding part of the TFF derivative, $\int_{0}^{x} \bar{\rho}_{n}\left(Q^{2}, t\right) \frac{t}{(\bar{t})^{2}} d t$, Eq. (A22), we have to integrate these expressions by inserting for $\bar{\rho}_{n}$ Eqs. A44. Those terms that are proportional to $\psi_{n}(x)$ only, Eqs. A4a and (A4b), are obtained by the following closed-form expressions

$$
\begin{aligned}
I_{1 \mathrm{H}}(n, x)= & \int_{0}^{x} \psi_{n}(t) \frac{1}{(1-t)} d t \equiv 6 \int_{0}^{x} C_{n}^{(3 / 2)}(2 t-1) t d t=\frac{1}{2}\left\{x C_{n+1}^{(1 / 2)}(2 x-1)\right. \\
& \left.-\frac{1}{2(2 n+3)}\left[C_{n+2}^{(1 / 2)}(2 x-1)-C_{n}^{(1 / 2)}(2 x-1)\right]\right\} ; \\
J_{1 \mathrm{H}}(n, x)= & \int_{0}^{x} \psi_{n}(t) \frac{t}{(1-t)^{2}} d t \equiv 6 \int_{0}^{x} C_{n}^{(3 / 2)}(2 t-1) \frac{t^{2}}{(1-t)} d t=3\left\{(-1)^{n+1}-(n+1)(n+2) \ln (\bar{x})\right. \\
& \left.-(x+1) C_{n+1}^{(1 / 2)}(2 x-1)+\frac{1}{2(2 n+3)}\left[C_{n+2}^{(1 / 2)}(2 x-1)-C_{n}^{(1 / 2)}(2 x-1)\right]+S(n, 0)-S(n, x)\right\},
\end{aligned}
$$

where

$$
S(n, x)=\sum_{m=1}^{n} \frac{(n+m+2) !}{m !(m+1) !(n-m) !} \frac{(-1)^{m}}{m}(1-x)^{m} .
$$

The treatment of the first term of Eq. (A4c) of $\bar{\rho}_{n}^{(1)}$ demands some care (see [2] for the origin of this term and further details). The results for the TFF and its deriva- 
tive are given by

$$
\begin{aligned}
& \int_{0}^{x}\left[2 v(n) \ln \left(\frac{\bar{t}}{\bar{t}}\right)-\ln ^{2}\left(\frac{\bar{t}}{\bar{t}}\right)\right] \frac{\psi_{n}(t)}{\bar{t}} d t=6\left[\left(2 v(n) \ln \left(\frac{\bar{t}}{\bar{t}}\right)-\ln ^{2}\left(\frac{\bar{t}}{\bar{t}}\right)\right) t\right]_{+(x)} \otimes C_{n}^{(3 / 2)}(2 t-1)+ \\
& 6 C_{n}^{(3 / 2)}(2 x-1) \cdot I_{2 \mathrm{H}}(x), \\
& \int_{0}^{x}\left[2 v(n) \ln \left(\frac{\bar{t}}{\bar{t}}\right)-\ln ^{2}\left(\frac{\bar{t}}{\bar{t}}\right)\right] \frac{\psi_{n}(t) t}{(\bar{t})^{2}} d t=6\left[\left(2 v(n) \ln \left(\frac{\bar{t}}{\bar{t}}\right)-\ln ^{2}\left(\frac{\bar{t}}{\bar{t}}\right)\right) \frac{t^{2}}{\bar{t}}\right]_{+(x)} \otimes C_{n}^{(3 / 2)}(2 t-1)+ \\
& 6 C_{n}^{(3 / 2)}(2 x-1) \cdot J_{2 \mathrm{H}}(x),
\end{aligned}
$$

where $f(t)_{+(x)} \otimes g(t)=\int_{0}^{x} f(t)(g(t)-g(x)) d t$.

read

In these equations we have isolated the purely $x$ dependent terms in two closed-form expressions, which

$$
\begin{aligned}
I_{2 \mathrm{H}}(x)= & \int_{0}^{x}\left[2 v(n) \ln \left(\frac{\bar{t}}{\bar{t}}\right)-\ln ^{2}\left(\frac{\bar{t}}{\bar{t}}\right)\right] t d t= \\
= & -v(n)\left[\left(1-x^{2}\right) \ln (\bar{x})+x(x \ln (x)+1)\right]-\left(\mathrm{Li}_{2}(x)+x \ln (x)\right)- \\
& \frac{\ln ^{2}(x)}{2} x^{2}+\frac{\ln ^{2}(\bar{x})}{2}\left(1-x^{2}\right)-\bar{x}((1+x) \ln (x)+1) \ln (\bar{x}) \\
J_{2 \mathrm{H}}(x)= & \int_{0}^{x}\left[2 v(n) \ln \left(\frac{\bar{t}}{t}\right)-\ln ^{2}\left(\frac{\bar{t}}{\bar{t}}\right)\right] \frac{t^{2}}{\bar{t}} d t= \\
= & 2 v(n)\left[2 \mathrm{Li}_{2}(x)+x^{2} \ln (x)+\ln (\bar{x})\left(-x^{2}-2 x+2 \ln (x)+3\right)+x-\ln ^{2}(\bar{x})+2 x \ln (x)\right]+\ln (\bar{x}) \\
& \left(-2 \mathrm{Li}_{2}(x)+x^{2}(-\ln (x))-x+\ln ^{2}(x)-2 x \ln (x)+3 \ln (x)+\frac{\pi^{2}}{3}+1\right)+3 \operatorname{Li}_{2}(x)-2 \mathrm{Li}_{3}(\bar{x})-2 \mathrm{Li}_{3}(x)+ \\
& 2 \mathrm{Li}_{2}(x) \ln (x)+\left(\frac{x^{2}}{2}+x-2 \ln (x)-\frac{3}{2}\right) \ln ^{2}(\bar{x})+\frac{1}{2} x^{2} \ln ^{2}(x)+\frac{1}{3} \ln ^{3}(\bar{x})+x \ln ^{2}(x)+x \ln (x)+2 \zeta_{3}
\end{aligned}
$$

recalling that $v(n)$ and $v^{b}(n)$ are the eigenvalues of the LO ERBL equations. These two factors, $I_{2 \mathrm{H}}(x)$ and $J_{2 \mathrm{H}}$, prevail over the corresponding remainders for large $n$.

\section{Appendix C: Moments vs. coefficients}

The relation between the Gegenbauer coefficients $a_{2 n}$ and the moments $\left\langle\xi^{2 m}\right\rangle=\left\langle(1-2 x)^{2 m}\right\rangle,(m \leq n)$ is given by

$$
\begin{aligned}
a_{2 n}= & \frac{2}{3} \frac{(4 n+3)}{(2 n+1)(2 n+2) 2^{2 n}} \cdot \sum_{m=0}^{n}(-1)^{(n-m)} \\
& \times \frac{(2 n+2 m+1) !}{(n+m) !(n-m) !(2 m) !}\left\langle\xi^{2 m}\right\rangle
\end{aligned}
$$

with the following first three coefficients

$$
\begin{aligned}
& a_{2}=\frac{7}{12}\left(5\left\langle\xi^{2}\right\rangle-1\right) \\
& a_{4}=\frac{77}{8}\left(\left\langle\xi^{4}\right\rangle-\frac{2}{3}\left\langle\xi^{2}\right\rangle+\frac{1}{21}\right) \\
& a_{6}=\frac{5}{64}\left(429\left\langle\xi^{6}\right\rangle-495\left\langle\xi^{4}\right\rangle+135\left\langle\xi^{2}\right\rangle-5\right)
\end{aligned}
$$

[1] B. Aubert et al. (The BaBar), Phys. Rev. D80, 052002 (2009), 0905.4778.

[2] S. V. Mikhailov and N. G. Stefanis, Nucl. Phys. B821, 
291 (2009), 0905.4004.

[3] S. J. Brodsky and G. P. Lepage, Adv. Ser. Direct. High Energy Phys. 5, 93 (1989).

[4] A. P. Bakulev, S. V. Mikhailov, A. V. Pimikov, and N. G. Stefanis, Phys. Rev. D84, 034014 (2011), 1105.2753.

[5] N. Stefanis, A. P. Bakulev, S. Mikhailov, and A. Pimikov, Nucl. Phys. Proc. Suppl. 225-227, 146 (2012), 1111.7137.

[6] S. S. Agaev, V. M. Braun, N. Offen, and F. A. Porkert, Phys. Rev. D83, 054020 (2011), 1012.4671.

[7] A. P. Bakulev, S. V. Mikhailov, and N. G. Stefanis, Phys. Lett. B508, 279 (2001), hep-ph/0103119.

[8] S. Uehara et al. (Belle Collaboration), Phys. Rev. D86, 092007 (2012), 1205.3249.

[9] H. J. Behrend et al. (CELLO), Z. Phys. C49, 401 (1991).

[10] J. Gronberg et al. (CLEO), Phys. Rev. D57, 33 (1998), hep-ex/9707031.

[11] A. P. Bakulev, S. V. Mikhailov, A. V. Pimikov, and N. G. Stefanis, Phys. Rev. D86, 031501 (2012), 1205.3770.

[12] A. Pimikov, A. Bakulev, S. Mikhailov, and N. Stefanis, AIP Conf. Proc. 1492, 134 (2012), 1208.4754.

[13] A. Bakulev, S. Mikhailov, A. Pimikov, and N. Stefanis, Acta Phys. Polon. Supp. 6, 137 (2013), 1212.0644.

[14] A. P. Bakulev, S. V. Mikhailov, and N. G. Stefanis, Phys. Lett. B578, 91 (2004), hep-ph/0303039.

[15] A. P. Bakulev, S. V. Mikhailov, and N. G. Stefanis, Phys. Rev. D73, 056002 (2006), hep-ph/0512119.

[16] S. V. Mikhailov and A. V. Radyushkin, JETP Lett. 43, 712 (1986).

[17] A. P. Bakulev and A. V. Radyushkin, Phys. Lett. B271, 223 (1991).

[18] P. del Amo Sanchez et al. (BABAR Collaboration), Phys. Rev. D84, 052001 (2011), 1101.1142.

[19] T. Feldmann, P. Kroll, and B. Stech, Phys. Rev. D58, 114006 (1998), hep-ph/9802409.

[20] A. V. Radyushkin, Phys. Rev. D80, 094009 (2009), 0906.0323.

[21] M. V. Polyakov, JETP Lett. 90, 228 (2009), 0906.0538.

[22] A. P. Bakulev, S. V. Mikhailov, and N. G. Stefanis, Phys. Rev. D67, 074012 (2003), hep-ph/0212250.

[23] A. P. Bakulev, S. V. Mikhailov, A. V. Pimikov, and N. G. Stefanis, Nucl. Phys. B (Proc. Suppl.) 219-220, 133 (2011), 1108.4344 .

[24] S. Agaev, V. Braun, N. Offen, and F. Porkert, Phys. Rev. D86, 077504 (2012), 1206.3968.

[25] A. Khodjamirian, Eur. Phys. J. C6, 477 (1999), hep$\mathrm{ph} / 9712451$

[26] A. P. Bakulev, S. V. Mikhailov, and N. G. Stefanis, Annalen Phys. 13, 629 (2004), hep-ph/0410138.

[27] A. P. Bakulev and S. V. Mikhailov, Phys. Rev. D65, 114511 (2002), hep-ph/0203046.

[28] N. G. Stefanis, W. Schroers, and H.-C. Kim, Phys. Lett. B449, 299 (1999), hep-ph/9807298.

[29] V. L. Chernyak and A. R. Zhitnitsky, Phys. Rept. 112, 173 (1984).

[30] B. Melić, D. Müller, and K. Passek-Kumerički, Phys. Rev. D68, 014013 (2003), hep-ph/0212346.

[31] H. Czyż, S. Ivashyn, A. Korchin, and O. Shekhovtsova, Phys. Rev. D85, 094010 (2012), 1202.1171.

[32] A. P. Bakulev and S. V. Mikhailov, Phys. Lett. B436, 351 (1998), hep-ph/9803298.

[33] S. L. Adler, Phys. Rev. 177, 2426 (1969).

[34] J. S. Bell and R. Jackiw, Nuovo Cim. A60, 47 (1969).

[35] S. J. Brodsky and G. P. Lepage, Phys. Rev. D24, 1808
(1981).

[36] E. Ruiz Arriola and W. Broniowski, Phys. Rev. D66, 094016 (2002), hep-ph/0207266.

[37] E. Ruiz Arriola and W. Broniowski, Phys. Rev. D67, 074021 (2003), hep-ph/0301202.

[38] V. Y. Petrov, M. V. Polyakov, R. Ruskov, C. Weiss, and K. Goeke, Phys. Rev. D59, 114018 (1999), hep$\mathrm{ph} / 9807229$.

[39] I. Anikin, A. Dorokhov, and L. Tomio, Phys. Lett. B475, 361 (2000), hep-ph/9909368.

[40] A. E. Dorokhov, Phys. Part. Nucl. Lett. 7, 229 (2010), 0905.4577.

[41] A. E. Dorokhov (2011), 1109.3754, 1109.3754.

[42] H.-n. Li and S. Mishima, Phys. Rev. D80, 074024 (2009), 0907.0166.

[43] E. R. Arriola and W. Broniowski, Phys. Rev. D81, 094021 (2010), 1004.0837.

[44] S. Noguera and V. Vento, Eur. Phys. J. A46, 197 (2010), 1001.3075

[45] X.-G. Wu and T. Huang, Phys. Rev. D82, 034024 (2010), 1005.3359.

[46] P. Kroll, Eur. Phys. J. C71, 1623 (2011), 1012.3542.

[47] X.-G. Wu and T. Huang, Phys. Rev. D84, 074011 (2011), 1106.4365 .

[48] T. N. Pham and X. Y. Pham, Int. J. Mod. Phys. A26, 4125 (2011), 1101.3177.

[49] C.-C. Lih and C.-Q. Geng, Phys. Rev. C85, 018201 (2012), 1201.2220.

[50] Z.-k. Guo and Q. Zhao, Eur. Phys. J. C72, 1964 (2012), 1108.0241 .

[51] I. Balakireva, W. Lucha, and D. Melikhov, Phys. Rev. D85, 036006 (2012), 1110.6904.

[52] Y. Klopot, A. Oganesian, and O. Teryaev, JETP Lett. 94, 729 (2011), 1110.0474.

[53] F. Zuo and T. Huang, Eur. Phys. J. C72, 1813 (2011), 1105.6008.

[54] T. Feldmann, Int. J. Mod. Phys. A15, 159 (2000), hepph/9907491.

[55] S. Noguera and S. Scopetta, Phys. Rev. D85, 054004 (2012), 1110.6402.

[56] Y. Klopot, A. Oganesian, and O. Teryaev, Phys. Rev. D87, 036013 (2013), 1211.0874.

[57] Y. Klopot, A. Oganesian, and O. Teryaev, Acta Phys. Polon. Supp. 6, 145 (2013), 1212.0459.

[58] M. Unverzagt, J. Phys. Conf. Ser. 349, 012015 (2012).

[59] H. L. L. Roberts, C. D. Roberts, A. Bashir, L. X. Gutierrez-Guerrero, and P. C. Tandy, Phys. Rev. C82, 065202 (2010), 1009.0067.

[60] H. R. Grigoryan and A. V. Radyushkin, Phys. Rev. D78, 115008 (2008), 0808.1243.

[61] S. J. Brodsky, F.-G. Cao, and G. F. de Teramond, Phys. Rev. D84, 033001 (2011), 1104.3364.

[62] S. J. Brodsky, G. de Teramond, and M. Karliner, Ann. Rev. Nucl. Part. Sci. 62, 1 (2012), 1302.5684.

[63] A. V. Efremov and A. V. Radyushkin, Phys. Lett. B94, 245 (1980).

[64] A. V. Efremov and A. V. Radyushkin, Theor. Math. Phys. 42, 97 (1980).

[65] G. P. Lepage and S. J. Brodsky, Phys. Rev. D22, 2157 (1980).

[66] W. Broniowski and E. R. Arriola, in Proceedings of the Mini-Workshop "Problems in Multi-Quark States", Bled, Slovenia, June 29-July 6, 2009, edited by B. Golli, M. Rosina, and S. Sirca (University of Ljubljana and 
Jozef Stefan Institute, Ljubljana, 2009), pp. 20-27, 0910.0869.

[67] P. Lichard, Phys. Rev. D83, 037503 (2011), 1012.5634.

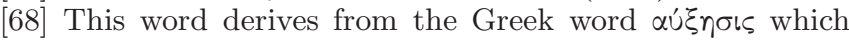
means the inherent tendency to increase. Auxetic materials have a negative Poisson's ratio and increase their cross section perpendicular to the applied force when stretched. That behavior is considered as an oddity.

[69] We thank Wojciech Broniowski for attracting our atten- tion to this point (see in this context also [43, 66 ]). The dependence of the $\pi^{0}$ TFF on the virtuality of the quasireal photon up to was recently discussed in [67], using the vector-meson-dominance hypothesis.

[70] The value $q^{2} \simeq 0.04 \mathrm{GeV}^{2}$ is due to $\mathrm{S}$. Uehara, private communication during the Meson TFF Workshop, 29-30 May, 2012, Cracow, Poland.

[71] Marc Unverzagt, private communication. 